\title{
Fast Predictions of Variance Images for Fan-Beam Transmission Tomography With Quadratic Regularization
}

\author{
Yingying Zhang-O'Connor, Student Member, IEEE, and Jeffrey A. Fessler, Fellow, IEEE
}

\begin{abstract}
Accurate predictions of image variances can be useful for reconstruction algorithm analysis and for the design of regularization methods. Computing the predicted variance at every pixel using matrix-based approximations [1] is impractical. Even most recently adopted methods that are based on local discrete Fourier approximations are impractical since they would require a forward and backprojection and two fast Fourier transform (FFT) calculations for every pixel, particularly for shift-variant systems like fan-beam tomography. This paper describes new "analytical" approaches to predicting the approximate variance maps of 2-D images that are reconstructed by penalized-likelihood estimation with quadratic regularization in fan-beam geometries. The simplest of the proposed analytical approaches requires computation equivalent to one backprojection and some summations, so it is computationally practical even for the data sizes in X-ray computed tomography (CT). Simulation results show that it gives accurate predictions of the variance maps. The parallel-beam geometry is a simple special case of the fan-beam analysis. The analysis is also applicable to 2-D positron emission tomography (PET).
\end{abstract}

Index Terms-Fan-beam tomography, local discrete Fourier analysis, penalized-likelihood image reconstruction, variance approximation.

\section{INTRODUCTION}

$\mathbf{S}$ TATISTICAL methods have obtained increasing attention in tomographic image reconstruction due to improved noise and resolution properties. These methods are usually nonlinear and shift-variant. To analyze the statistical characteristics of the reconstructed images, one would like to be able to predict the variances and covariances of estimated pixel values. The variance information provides an uncertainty measure of the reconstructed image and may aid regularization parameter selection.

The existing noise analysis methods can be divided into two categories: iteration based and estimator based. The iteration-based variance predictions are studied in e.g., [2] and [3] as a function of the iteration number for the maximum-likelihood expectation maximization algorithm based on the "stopping rule" to terminate the iterations before convergence. The

Manuscript received July 10, 2006; revised October 11, 2006. This work was supported in part by the National Institutes of Health/National Cancer Institute (NIH/NCI) under Grant P01 CA87634 and in part by GE Medical Systems. Asterisk indicates corresponding author.

*Y. Zhang-O'Connor is with the the Electrical Engineering and Computer Science Department, The University of Michigan, Ann Arbor, MI 48109 USA (e-mail: yzz@eecs.umich.edu).

J. A. Fessler is with the Electrical Engineering and Computer Science Department, The University of Michigan, Ann Arbor, MI 48109 USA (e-mail: fessler@eecs.umich.edu).

Digital Object Identifier 10.1109/TMI.2006.887368 estimator-based variance predictions are independent of the particular algorithm and iterations, [1], [4], [5]. Our proposed method falls in the estimator-based category. We will give a brief overview on the existing estimator-based methods and our proposed method.

The estimator-based analysis for the mean and variance proposed in [1] uses the partial derivatives of the cost function and Taylor approximations. The approximations are in matrix form and give accurate results. However, the predictions involve the inversion of the Hessian matrices and therefore are computationally expensive. Based on this work, a great deal of effort has been given to simplify these matrix methods [4], [5]. All these methods, that we refer to the discrete Fourier transform (DFT) approximations, are based on a factorization of the system matrix and circulant approximations to the Hessian matrices to precompute and store a great portion of the calculations. The factorization of the system matrix into geometric and object-dependent portions is specially useful for the shift-varying imaging systems. However, these DFT approximations still require in precomputation one forward and backprojection and two fast Fourier transform (FFT) calculations, one for likelihood Hessian and one for penalty Hessian, for each location of interest. Moreover, the expressions are still in matrix form and provide little direct insight into the noise properties.

Our proposed approximations in this paper are still based on the results given in [1], but turn to a very different analysis approach. Instead of working in the discrete space, we use the discrete space Fourier transform (DSFT) and Parseval's theorem to bridge from the discrete space to the continuous space. Using local shift-invariance approximations and local Fourier analysis, we derive "analytical" closed-form expressions for the local impulse response and local frequency response of the Gram operator and the regularization operator. The final approximations eliminate the need of FFTs for variance predictions, greatly reducing computation for cases where the variance is to be predicted at numerous pixel locations. Furthermore, these approximations provide insight into the resolution and noise properties of the reconstructed images.

Because our analysis is built on the previous work in [1], we briefly repeat its main results here. The goal of transmission image reconstruction is to estimate an attenuation image $\mu[\vec{n}]$ from projection data $\boldsymbol{Y}$, where $\vec{n}$ is a vector denoting the 2-D image pixel location. We focus here on penalized-likelihood estimators obtained by minimizing a cost function as follows:

$$
\hat{\boldsymbol{\mu}}=\arg \min _{\boldsymbol{\mu}} \Phi(\boldsymbol{\mu}, \boldsymbol{Y})
$$




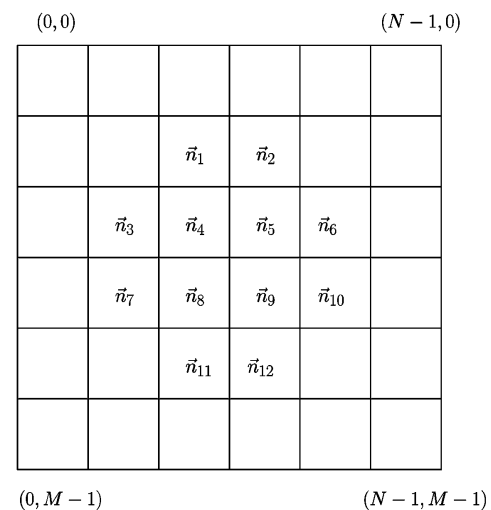

Fig. 1. A $N \times M$ lattice with approximately circular FOV. Only the pixels with indices are estimated. In this example, $p=|\mathcal{S}|=12$.

where $\boldsymbol{\mu}=\left(\mu\left[\vec{n}_{1}\right], \ldots, \mu\left[\vec{n}_{p}\right]\right) \in \mathbb{R}^{p}$ ( $p$-dimensional real space). The cost function includes a negative log-likelihood term and a regularization term

$$
\Phi(\boldsymbol{\mu}, \boldsymbol{Y})=-L(\boldsymbol{\mu}, \boldsymbol{Y})+\alpha R(\boldsymbol{\mu}) .
$$

As a concrete example, for transmission tomography under the Poisson noise model, the log-likelihood is

$$
L(\boldsymbol{\mu}, \boldsymbol{Y})=\sum_{i} Y_{i} \log \left(\bar{Y}_{i}(\boldsymbol{\mu})\right)-\bar{Y}_{i}(\boldsymbol{\mu}) .
$$

For mono-energetic transmission scans, the measurement means are modeled by

$$
\bar{Y}_{i}(\boldsymbol{\mu})=b_{i} \mathrm{e}^{-[\boldsymbol{A} \boldsymbol{\mu}]_{i}}+r_{i}
$$

where $A$ is the system matrix, $b_{i}$ denotes the blank scan, and $r_{i}$ denotes the additive contribution of scatter to the $i$ th ray.

We focus on regularization terms of the following form:

$$
\begin{aligned}
R(\boldsymbol{\mu}) & =\sum_{l=1}^{4} R_{l}(\boldsymbol{\mu}) \\
R_{l}(\boldsymbol{\mu}) & =\sum_{\vec{n}, \vec{n}-\vec{m}_{l} \in \mathcal{S}} r_{l}[\vec{n}] \frac{1}{2}\left(\mu[\vec{n}]-\mu\left[\vec{n}-\vec{m}_{l}\right]\right)^{2}
\end{aligned}
$$

where $\mathcal{S} \triangleq\left\{\vec{n}_{j}: j=1, \ldots, p\right\}$ denotes the subset of the $N \times M$ lattice that is estimated and $\vec{m}_{l} \in\{(1,0),(0,1),(1,1),(-1,1)\}$. The roughness penalty (4) involves the horizontal, vertical, and diagonal neighbors and allows for the possibility of using regularization coefficients $\left\{r_{l}[\vec{n}]\right\}$ that vary both with spatial location and direction [6], [7]. In general, $1 \leq p \leq N M$ and $p<N M$ because the physical field-of-view (FOV) is a subset of the lattice (see Fig. 1).

The goal of this work is to approximate the covariance matrix $\operatorname{Cov}\{\hat{\boldsymbol{\mu}}\}$ efficiently yet accurately, motivated by the problem of designing the regularizer $R(\boldsymbol{\mu})$. The proposed prediction methods can be generalized to other log-likelihood terms including 2-D emission tomography by modifying $W$ in (6).

The following approximation to the $p \times p$ covariance matrix of $\hat{\boldsymbol{\mu}}$ was derived in [1]:

$$
\boldsymbol{K}=\left(\boldsymbol{A}^{\prime} \boldsymbol{W} \boldsymbol{A}+\alpha \mathbf{P}\right)^{-1} \boldsymbol{A}^{\prime} \boldsymbol{W} \boldsymbol{A}\left(\boldsymbol{A}^{\prime} \boldsymbol{W} \boldsymbol{A}+\alpha \mathbf{P}\right)^{-1}
$$

where $\mathbf{P}$ is the Hessian matrix of the roughness penalty. For transmission tomography with the models (1) and (2), $\boldsymbol{W}=$ $\operatorname{diag}\left\{\bar{Y}_{i}\right\}$. In practice $\bar{Y}_{i}$ is unknown, so we plug in $Y_{i}$ as an approximation [8]. The covariance between pixels $\hat{\mu}\left[\vec{n}_{k}\right]$ and $\hat{\mu}\left[\vec{n}_{j}\right]$ can be approximated using (6) as follows:

$$
\operatorname{Cov}\left\{\hat{\mu}\left[\vec{n}_{j}\right], \hat{\mu}\left[\vec{n}_{k}\right]\right\} \approx \mathbf{e}_{j}^{\prime} \boldsymbol{K} \mathbf{e}_{k}
$$

where $\mathbf{e}_{j}$ denotes the $j$ th unit column vector of length $p$.

The matrix method described in (6) and (7) has been used in various applications [5], [9]. Simulation and experimental results have confirmed the accuracy of this covariance approximation in image regions where the nonnegativity constraint is usually inactive. However, evaluating (7) is relatively expensive. In this paper, we introduce "continuous space analysis" and use "local stationarity" to develop fast approximations for the variance and covariance of the reconstructed image $\hat{\mu}[\vec{n}]$.

The paper is organized as follows. Section II briefly reviews the matrix method and the local shift-invariance approximations. Section III proposes the general analytical approach for the variance approximation. Sections IV and V apply this method to fan-beam geometry and quadratic regularization. Sections VI and VII analyze the single integral approach used and give simulation results for two types of quadratic regularization, including a comparison of the predicted, DFT-based and empirical standard deviation images. Finally, discussion and conclusions are in Section VIII.

\section{LOCAL SHIFT-INVARIANCE APPROXIMATIONS}

The matrix method described in (6) and (7) is very expensive to compute, even for the variance at a single pixel. To accelerate computation, local shift-invariance approximations are usually used in practice, (e.g., [4], [5], and [9]-[11] ).

Let $\boldsymbol{M}$ denote one of the $p \times p$ matrices in (6), such as $\boldsymbol{A}^{\prime} \boldsymbol{W} \boldsymbol{A}$ or $\mathbf{P}$, or inverses or sums thereof. Then a matrix-vector operation $\boldsymbol{y}=\boldsymbol{M} \boldsymbol{x}$ can be expressed equivalently as

$$
\begin{aligned}
y[\vec{n}] & =\delta_{\mathcal{S}}[\vec{n}] \sum_{\vec{n}^{\prime} \in \mathcal{S}} h\left(\vec{n}, \vec{n}^{\prime}\right) x\left[\vec{n}^{\prime}\right] \\
& =\delta_{\mathcal{S}}[\vec{n}] \sum_{\vec{n}^{\prime}} h\left(\vec{n}, \vec{n}^{\prime}\right) x\left[\vec{n}^{\prime}\right] \delta_{\mathcal{S}}\left[\vec{n}^{\prime}\right]
\end{aligned}
$$

where $\delta_{\mathcal{S}}[\vec{n}]$ is an indicator function of $\vec{n}$ defined as follows:

$$
\delta_{\mathcal{S}}[\vec{n}] \triangleq\left\{\begin{array}{ll}
1, & \vec{n} \in \mathcal{S} \\
0, & \text { otherwise }
\end{array} .\right.
$$

In other words, the elements of $\boldsymbol{M}$ correspond to $M_{k j}=$ $h\left(\vec{n}_{k}, \vec{n}_{j}\right)$.

Near a given location $\vec{n}_{0}$ of interest, we define a local impulse response of $\boldsymbol{M}$ as follows 1 :

$$
\begin{aligned}
h_{0}(\vec{m}) \triangleq h\left(\vec{n}_{0}+\lambda \vec{m}, \vec{n}_{0}-(1-\lambda) \vec{m}\right) \\
\quad \delta_{\mathcal{S}}\left[\vec{n}_{0}+\lambda \vec{m}\right] \delta_{\mathcal{S}}\left[\vec{n}_{0}-(1-\lambda) \vec{m}\right]
\end{aligned}
$$

\footnotetext{
${ }^{1}$ Throughout the paper we use the subscript " 0 " to indicate dependence on a given pixel location $\vec{n}_{0}$.
} 
where $\vec{m} \in \mathbb{Z}^{2}, \mathbb{Z}$ denotes the set of integers. Usually we choose $\lambda=1$. However, sometimes we can approximate $h$ even for non-integer arguments, in which case $\lambda=1 / 2$ may also be useful [12, p. 870].

We say that $h\left(\vec{n}, \vec{n}^{\prime}\right)$ is locally shift invariant near $\vec{n}_{0}$ if $h\left(\vec{n}, \vec{n}^{\prime}\right) \approx h_{0}\left(\vec{n}-\vec{n}^{\prime}\right)$ for $\vec{n}$ and $\vec{n}^{\prime}$ close to $\vec{n}_{0}$. The approximation should be accurate provided $\vec{n}$ and $\vec{n}^{\prime}$ are "sufficiently close" to $\vec{n}_{0}$ relative to the width of $h_{0}$. Thus, if the operator $\boldsymbol{M}$ is approximately locally shift invariant near $\vec{n}_{0}$, then we can approximate the superposition sum (8) by (almost) a convolution sum

$$
y[\vec{n}] \approx \delta_{\mathcal{S}}[\vec{n}] \sum_{\vec{n}^{\prime}} h_{0}\left(\vec{n}-\vec{n}^{\prime}\right) x\left[\vec{n}^{\prime}\right] \delta_{\mathcal{S}}\left[\vec{n}^{\prime}\right]
$$

or equivalently $\boldsymbol{y} \approx \boldsymbol{M}_{0} \boldsymbol{x}$, where the $p \times p$ matrix $\boldsymbol{M}_{0}$ is defined by $\left[\boldsymbol{M}_{0}\right]_{k j}=h_{0}\left(\vec{n}_{k}-\vec{n}_{j}\right)$. The expression (11) is almost a convolution sum, except for the "edge conditions" of the indicator functions. If the point $\vec{n}_{0}$ is not "too close" to the boundaries of the support mask $\mathcal{S}$, then we may able to disregard the indicator functions and treat the expression as a convolution.

Let $\mathbf{T}$ be the $N M \times p$ matrix such that

$$
\mathbf{T}_{1+n+m N, j}= \begin{cases}1, & \vec{n}_{j}=(n, m) \\ 0, & \text { otherwise }\end{cases}
$$

for $n=0, \ldots N-1$ and $m=0, \ldots M-1$. The purpose of $\mathbf{T}$ is to embed the $p$ elements of $\boldsymbol{\mu}$ (as shown in Fig. 1) back to the 2-D $N \times M$ lattice. Then $\boldsymbol{M}_{0}=\mathbf{T}^{\prime} \breve{\boldsymbol{M}}_{0} \mathbf{T}$, where $\left(\breve{\boldsymbol{M}}_{0}\right)_{\vec{n}, \vec{n}^{\prime}}=h_{0}\left(\vec{n}-\vec{n}^{\prime}\right)$ is an $N M \times N M$ matrix that is block Toeplitz with Toeplitz blocks BTTB. Thus, we can make a circulant approximation to $\breve{\boldsymbol{M}}_{0}$, (see [13]). Such approximations are often reasonably accurate except near the edges of the FOV, where the differences between "Toeplitz" and "circulant" end conditions are largest. The local impulse response (10) and the corresponding circulant approximation are two key tools for analysis.

\section{The Analytical Variance PRediction}

In the spirit of the local shift-invariance approximations presented in Section II, we approximate the covariance matrix in (6) near a given location $\vec{n}_{0}$ by

$$
\begin{aligned}
& \boldsymbol{K} \approx \boldsymbol{K}_{0} \triangleq \mathbf{T}^{\prime} \breve{\boldsymbol{K}}_{0} \mathbf{T} \\
& \breve{\boldsymbol{K}}_{0} \triangleq\left(\mathbf{F}_{0}+\alpha \mathbf{P}_{0}\right)^{-1} \mathbf{F}_{0}\left(\mathbf{F}_{0}+\alpha \mathbf{P}_{0}\right)^{-1}
\end{aligned}
$$

where $\mathbf{F}_{0}$ and $\mathbf{P}_{0}$ are the $N M \times N M$ BTTB approximations corresponding to $\boldsymbol{A}^{\prime} \boldsymbol{W} \boldsymbol{A}$ and $\mathrm{P}$, respectively. Then we approximate the covariance between pixels $\hat{\mu}[\vec{n}]$ and $\hat{\mu}\left[\vec{n}^{\prime}\right]$ in (7) by the following inner product:

$$
\operatorname{Cov}\left\{\hat{\mu}[\vec{n}], \hat{\mu}\left[\vec{n}^{\prime}\right]\right\} \approx\left\langle\breve{K}_{0} \mathbf{e}_{\vec{n}^{\prime}}, \mathbf{e}_{\vec{n}}\right\rangle
$$

where $\mathbf{e}_{\vec{n}}$ is $\vec{n}$ th unit vector of length $N M$.

Two useful approximations to (12) follow from Parseval's theorem. One option is to interpret the arguments in (11) with a suitable modulo $N$ or $M$. In this case, the inner product defined in (12) is in the form of circulant convolution and can be approximated by FFTs

$$
\operatorname{Cov}\left\{\hat{\mu}[\vec{n}], \hat{\mu}\left[\vec{n}^{\prime}\right]\right\} \approx \frac{1}{N M} \sum_{\vec{k}=\overrightarrow{0}}^{\vec{N}-1} P_{\mathrm{d} 0}[\vec{k}] e^{i \vec{\omega}_{\vec{k}} \cdot\left(\vec{n}-\vec{n}^{\prime}\right)}
$$

for $\vec{n}, \vec{n}^{\prime} \approx \vec{n}_{0}$, where $\vec{N}=(N, M), \vec{\omega}_{\vec{k}}=\left(2 \pi k_{1} / N, 2 \pi k_{2} / M\right)$ and

$$
P_{\mathrm{d} 0}[\vec{k}] \triangleq \frac{\Gamma_{0}[\vec{k}]}{\left(\Gamma_{0}[\vec{k}]+\alpha \Omega_{0}[\vec{k}]\right)^{2}}
$$

with

$$
\begin{aligned}
\mathbf{F}_{0} & \approx Q \Gamma_{0} Q^{\prime} \\
\mathbf{P}_{0} & \approx Q \boldsymbol{\Omega}_{0} Q^{\prime}
\end{aligned}
$$

where $\boldsymbol{Q}$ is the 2-D $(N, M)$-point orthonormal DFT matrix. The diagonal matrices $\boldsymbol{\Gamma}_{0}$ and $\boldsymbol{\Omega}_{0}$ have diagonal elements $\Gamma_{0}[\vec{k}]$ and $\Omega_{0}[\vec{k}]$ that are the 2-D DFT coefficients of the local impulse response of $\boldsymbol{A}^{\prime} \boldsymbol{W} \boldsymbol{A}$ and $\mathbf{P}$ near $\vec{n}_{0}$, respectively. This DFT/FFT approximation has been used in [4], [14], and [15] to predict variance at a single pixel

$$
\begin{aligned}
\operatorname{Var}\left\{\hat{\mu}\left[\vec{n}_{0}\right]\right\} & \approx\left\langle\breve{\boldsymbol{K}}_{0} \mathbf{e}_{\vec{n}_{0}}, \mathbf{e}_{\vec{n}_{0}}\right\rangle \\
& \approx \frac{1}{N M} \sum_{\vec{k}=0}^{\vec{N}-1} \frac{\Gamma_{0}[\vec{k}]}{\left(\Gamma_{0}[\vec{k}]+\alpha \Omega_{0}[\vec{k}]\right)^{2}} .
\end{aligned}
$$

Generally, evaluating this expression for a single pixel requires a forward and backprojection and two FFTs. Computation of this DFT approximation is still expensive for realistic image sizes when the variance must be computed for many or all pixels, particularly for shift-variant systems like fan-beam tomography.

An alternative option is to consider $\mu[\vec{n}]$ to be defined over all of $\mathbb{Z}^{2}$ (two-dimensional integer space), in which case (12) is in the form of ordinary convolution that can be expressed using the DSFT as follows:

$$
\operatorname{Cov}\left\{\hat{\mu}[\vec{n}], \hat{\mu}\left[\vec{n}^{\prime}\right]\right\} \approx \int_{-\pi}^{\pi} \int_{-\pi}^{\pi} P_{\mathrm{d} 0}(\vec{\omega}) e^{i \vec{\omega} \cdot\left(\vec{n}-\vec{n}^{\prime}\right)} \frac{\mathrm{d} \vec{\omega}}{(2 \pi)^{2}}
$$

where $P_{\mathrm{d} 0}(\vec{\omega})$ is the local spectrum of $\breve{\boldsymbol{K}}_{0}$, given as follows:

$$
P_{\mathrm{d} 0}(\vec{\omega}) \triangleq \frac{H_{\mathrm{d} 0}(\vec{\omega})}{\left[H_{\mathrm{d} 0}(\vec{\omega})+\alpha R_{\mathrm{d} 0}(\vec{\omega})\right]^{2}}
$$

where $H_{\mathrm{d} 0}(\vec{\omega})$ is the local frequency response of the Gram matrix $A^{\prime} \boldsymbol{W} \boldsymbol{A}$ and $R_{\mathrm{d} 0}(\vec{\omega})$ is the local frequency response of $\mathbf{P}$ near $\vec{n}_{0}$. To our knowledge, this paper is the first to use (15) to develop analytical variance approximations as a faster alternative to the DFT approach (14).

For regularizer design, the standard deviation map of the reconstructed image is one quantity of interest, and our numerical investigation will focus on variance prediction. However, the methodology applies readily to approximate covariances. 
Using the DSFT approximation (15), we approximate the variance at pixel $\vec{n}_{0}$ as follows:

$$
\operatorname{Var}\left\{\hat{\mu}\left[\vec{n}_{0}\right]\right\} \approx \int_{-\pi}^{\pi} \int_{-\pi}^{\pi} P_{\mathrm{d} 0}(\vec{\omega}) \frac{\mathrm{d} \vec{\omega}}{(2 \pi)^{2}} .
$$

Let $\Delta$ denote the sample spacing in the reconstructed image. By making the change of variable, $\vec{\omega}=(2 \pi \rho \Delta) \overrightarrow{e_{\Phi}}$ where $\vec{e}_{\Phi} \triangleq$ $(\cos \Phi, \sin \Phi)$, we rewrite (17) in terms of polar frequency coordinates $(\rho, \Phi)$ as follows:

$$
\operatorname{Var}\left\{\hat{\mu}\left[\vec{n}_{0}\right]\right\} \approx \Delta^{2} \int_{0}^{2 \pi} \int_{0}^{\rho_{\max }(\Phi)} P_{0}(\rho, \Phi) \rho \mathrm{d} \rho \mathrm{d} \Phi
$$

where $\rho_{\max }(\Phi)=1 /(2 \Delta \max \{|\cos \Phi|,|\sin \Phi|\})$, and we define

$$
P_{0}(\rho, \Phi) \triangleq P_{\mathrm{d} 0}\left(2 \pi \rho \Delta \vec{e}_{\Phi}\right)=\frac{H_{0}(\rho, \Phi)}{\left[H_{0}(\rho, \Phi)+\alpha R_{0}(\rho, \Phi)\right]^{2}} .
$$

We defined $H_{0}$ and $R_{0}$ similarly in terms of $H_{\mathrm{d} 0}$ and $R_{\mathrm{d} 0}$. The variance prediction (18) applies to any 2-D geometry. The Section IV specializes (18) by finding analytical approximations to the local frequency response $H_{0}(\rho, \Phi)$ for the fan-beam geometry.

\section{FAN-BEAM GEOMETRY}

The following analysis is focused on equiangular fan-beam transmission tomography with an arc detector. However, the method generalizes readily to flat detectors, i.e., equidistant sampling and to parallel-beam geometries. As illustrated in Fig. 2, fan-beam rays are indexed by coordinates $(s, \beta)$, where $\beta$ is the angle of the source relative to the $y$ axis, and $s$ is the arc length along the detector. For the case where the detector focal point is at the source position, $\gamma(s)=s / D_{\text {sd }}$, where $\gamma$ is the angle of the ray relative to the source and $D_{\mathrm{sd}}$ is the source to detector distance. The relation between parallel-beam and fan-beam coordinates is [16]

$$
\begin{aligned}
r(s) & =D_{\mathrm{s} 0} \sin \gamma(s) \\
\varphi(s, \beta) & =\beta+\gamma(s)
\end{aligned}
$$

where $D_{\mathrm{s} 0}$ is the source-to-rotation center distance.

\section{A. Local Impulse Response}

To predict variance images in fan-beam transmission tomography using (18), we need to determine the local frequency response $H_{0}(\rho, \Phi)$, or equivalently $H_{\mathrm{d} 0}(\vec{\omega})$. We first find the local impulse response.

Consider the 2-D object model based on a common basis function $\chi(\vec{x})$ superimposed on a $N \times M$ Cartesian grid as follows:

$$
\mu(\vec{x})=\sum_{\vec{n} \in \mathcal{S}} \mu[\vec{n}] \chi\left(\frac{\vec{x}-\vec{x}_{c}[\vec{n}]}{\Delta}\right)
$$

where $\vec{x} \in \mathbb{R}^{2}$ denotes the 2-D coordinates of the continuous image space, and $\vec{x}_{c}[\vec{n}]$ denotes the center of the basis function. Typically

$$
\begin{aligned}
\vec{x}_{c}[\vec{n}] & =\left(\vec{n}-\vec{\omega}_{\vec{x}}\right) \Delta, \quad \vec{n} \in \mathcal{S} \\
\vec{\omega}_{\vec{x}} & =\frac{(\vec{N}-\overrightarrow{1})}{2}+\vec{c}_{\vec{x}},
\end{aligned}
$$

where $\vec{N}=(N, M)$ and the user-selectable parameter $\vec{c}_{\vec{x}}$ denotes an optional spatial offset for the image center.

For simplicity, we assume here that the detector blur $b(s)$ is locally shift invariant, independent of source position $\beta$, and acts only along the $s$ coordinate. Then we model the mean projections as follows:

$$
\bar{y}_{\beta}\left[s_{k}\right]=\int b\left(s_{k}-s^{\prime}\right) p_{\varphi\left(s^{\prime}, \beta\right)}\left(r\left(s^{\prime}\right)\right) \mathrm{d} s^{\prime}
$$

for $s_{k}=\left(k-w_{s}\right) \Delta_{s}$ and $k=1, \ldots, n_{s}$, where $\Delta_{s}$ is the sample spacing in $s, w_{s}$ is defined akin to $\vec{w}_{\vec{x}}$, and $p_{\varphi}(r)$ is the 2-D Radon transform of $\mu(\vec{x})$

$$
p_{\varphi}(r)=\int \mu(r \cos \varphi-\ell \sin \varphi, r \sin \varphi+\ell \cos \varphi) \mathrm{d} \ell .
$$

Substituting the basis expansion model in (22) for the object into the measurement model (23) and simplifying leads to the linear model

$$
\bar{y}_{\beta}\left[s_{k}\right]=\sum_{\vec{n} \in \mathcal{S}} a\left(s_{k}, \beta ; \vec{n}\right) \mu[\vec{n}]
$$

where the fan-beam system matrix elements are samples of the following fan-beam projection of a single basis function centered at $\vec{x}_{c}[\vec{n}]$

$$
\begin{aligned}
& a(s, \beta ; \vec{n})=\int b\left(s-s^{\prime}\right) \Delta \\
& \quad \cdot g\left(\frac{r\left(s^{\prime}\right)-r_{\varphi\left(s^{\prime}, \beta\right)}[\vec{n}]}{\Delta}, \varphi\left(s^{\prime}, \beta\right)\right) \mathrm{d} s^{\prime}
\end{aligned}
$$

where $g(\cdot, \varphi)$ is the Radon transform of $\chi(\vec{x})$ at angle $\varphi$ and

$$
r_{\varphi}[\vec{n}] \triangleq \vec{x}_{c}[\vec{n}] \cdot \vec{e}_{\varphi}
$$

with $\vec{e}_{\varphi} \triangleq(\cos \varphi, \sin \varphi)$.

Then the elements of the Gram matrix are given exactly by

$$
\begin{aligned}
h_{\mathrm{d}}\left[\vec{n} ; \vec{n}^{\prime}\right] & = \begin{cases}{\left[\boldsymbol{A}^{\prime} \boldsymbol{W} \boldsymbol{A}\right]_{j j^{\prime}},} & \vec{n}=\vec{n}_{j} \in \mathcal{S}, \vec{n}^{\prime}=\vec{n}_{j^{\prime}} \in \mathcal{S} \\
0, & \text { otherwise }\end{cases} \\
& =\breve{h} \mathrm{~d}\left[\vec{n} ; \vec{n}^{\prime}\right] \eta\left(\vec{x}_{c}[\vec{n}]\right) \eta\left(\vec{x}_{c}\left[\vec{n}^{\prime}\right]\right)
\end{aligned}
$$

where $\eta\left(\vec{x}_{c}[\vec{n}]\right) \triangleq 1_{\{\vec{n} \in \mathcal{S}\}}$

$$
\breve{h}_{\mathrm{d}}\left[\vec{n} ; \vec{n}^{\prime}\right] \triangleq \sum_{l=1}^{n_{A}} \sum_{k=1}^{n_{s}} w\left(s_{k}, \beta_{l}\right) a\left(s_{k}, \beta_{l} ; \vec{n}\right) a\left(s_{k}, \beta_{l} ; \vec{n}^{\prime}\right)
$$

and $w(s, \beta)$ denotes the weighting associated with $\boldsymbol{W}$ and $n_{A}$ denotes the number of samples of the source position $\beta$. To 


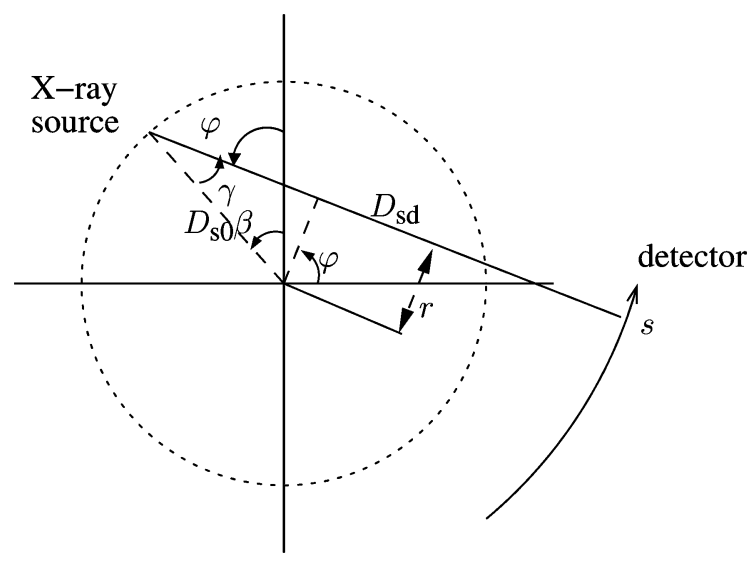

Fig. 2. Angular coordinates in fan-beam geometry.

simplify (25), we first use an integral to approximate the summation in (26) as follows:

$$
\begin{aligned}
\breve{h}_{\mathrm{d}}\left[\vec{n} ; \vec{n}^{\prime}\right] \approx \frac{1}{\Delta_{\beta}} \frac{1}{\Delta_{s}} \int_{0}^{2 \pi} \int_{-\infty}^{\infty} w(s, \beta) \\
\cdot a(s, \beta ; \vec{n}) a\left(s, \beta ; \vec{n}^{\prime}\right) \mathrm{d} s \mathrm{~d} \beta
\end{aligned}
$$

where $\Delta_{\beta}$ is the source angular sampling interval. Notice that $\breve{h} \breve{d}\left[\vec{n} ; \vec{n}^{\prime}\right]$ in (27) is not shift invariant.

We develop locally shift-invariant approximations to $\breve{h} \breve{\mathrm{d}}\left[\vec{n} ; \vec{n}^{\prime}\right]$ in (27) by reparameterizing variables $s, \beta$ using analogs of fan-to-parallel beam rebinning. The following locally shift-invariant approximation to $\breve{h} \mathrm{~d}\left[\vec{n} ; \vec{n}^{\prime}\right]$ is derived in detail in Appendix I:

$$
\breve{h}_{\mathrm{d}}\left[\vec{n} ; \vec{n}^{\prime}\right] \approx \frac{1}{\Delta_{\beta}} \frac{1}{\Delta_{s}} \int_{0}^{2 \pi} w_{0}(\varphi) \breve{h}_{0}\left(\Delta\left(\vec{n}-\vec{n}^{\prime}\right) \cdot \vec{e}_{\varphi}, \varphi\right) \mathrm{d} \varphi
$$

where the following 1-D autocorrelation is with respect to $r$

$$
\breve{h}_{0}(r, \varphi) \triangleq a_{0}(r, \varphi) \star a_{0}(r, \varphi)
$$

and $a_{0}(r, \varphi)$ is a locally parallel-beam version of the system model defined in (52) (see Appendix I). The angle-dependent weighting $w_{0}(\varphi)$ is associated with pixel $\vec{n}_{0}$, accounting for the position-dependent magnification as follows:

$$
\begin{aligned}
w_{0}(\varphi) & \triangleq\left|m_{0}(\varphi)\right| w\left(s\left(r_{0}(\varphi)\right), \beta\left(r_{0}(\varphi), \varphi\right)\right) \\
r_{0}(\varphi) & \triangleq r_{\varphi}\left[\vec{n}_{0}\right] \\
m_{0}(\varphi) & \left.\triangleq \frac{\partial}{\partial r} s(r)\right|_{r=r_{0}(\varphi)}=\frac{\frac{D_{\mathrm{sd}}}{D_{\mathrm{s} 0}}}{\sqrt{1-\left(\frac{r_{0}(\varphi)}{D_{\mathrm{s} 0}}\right)^{2}}}
\end{aligned}
$$

where $s(r)$ and $\beta(r, \varphi)$ are the inverse of (20) and (21). The shape of the local impulse response (28) is a modification of $1 / r$ (cf. [17]) with statistically modulated angular weighting. The key property of (28) is that it is locally shift invariant, except for edge effects. This approximation should be reasonably accurate provided that $\vec{n}$ and $\vec{n}^{\prime}$ are "sufficiently close" to $\vec{n}_{0}$, the coordinates of the pixel of interest.

\section{B. Local Frequency Response}

Having found the local impulse response approximation (28), the next step is to find the local frequency response. This requires consideration of the edge effects in (25).

The following local frequency response near a point $\vec{n}_{0}$ is derived in detail in Appendix II:

$$
H_{0}(\rho, \Phi) \approx \frac{1}{\Delta_{\beta} \Delta_{s} \Delta^{2}} \int_{0}^{2 \pi} w_{0}(\varphi) S_{\varphi}(\rho, \Phi) d \varphi
$$

where the following function captures both detector response effects and edge effects:

$$
\begin{aligned}
S_{\varphi}(\rho, \Phi)=\left|A_{0}(\rho \cos (\Phi-\varphi), \varphi)\right|^{2} \\
\cdot d_{0}(\varphi) \operatorname{sinc}^{2}\left(d_{0}(\varphi) \rho \sin (\Phi-\varphi)\right)
\end{aligned}
$$

$d_{0}(\varphi)$ denotes the length of the chord through $\vec{n}_{0}$ through the FOV at angle $(\varphi+\pi / 2)$, and $A_{0}(\nu, \varphi)$ is the 1-D Fourier transform (FT) of $a_{0}(r, \varphi)$ with respect to $r$.

\section{Further Approximations of Local Frequency Response}

The local frequency response of the Gram operator in (31) is very accurate. However, direct implementation of (31) is still computationally demanding. We present here two types of further approximations to simplify (31).

1) Type I Nonseparable Form: As $d_{0}(\varphi) \rightarrow \infty$, one can show that for large $|\rho|$

$$
d_{0}(\varphi) \operatorname{sinc}^{2}\left(d_{0}(\varphi) \rho \sin (\Phi-\varphi)\right) \rightarrow \delta(\rho \sin (\Phi-\varphi))
$$

Therefore, the $\operatorname{sinc}^{2}$ term is sharply peaked near $\Phi=\varphi$ and $\Phi=$ $\varphi \pm \pi$, so we consider the further simplifying approximation

$$
\int_{0}^{2 \pi} w_{0}(\varphi) S_{\varphi}(\rho, \Phi) \mathrm{d} \varphi \approx w_{0}(\Phi)\left|A_{0}(\rho, \Phi)\right|^{2} G_{0}(\rho, \Phi)
$$

where

$$
G_{0}(\rho, \Phi)=\int_{0}^{2 \pi} d_{0}(\varphi) \operatorname{sinc}^{2}\left(d_{0}(\varphi) \rho \sin (\Phi-\varphi)\right) \mathrm{d} \varphi .
$$

Substituting into (31) leads to the "Type I" approximation

$$
H_{0}(\rho, \Phi) \approx H_{01}(\rho, \Phi) \triangleq \frac{w_{0}(\Phi)}{\Delta_{\beta} \Delta_{s} \Delta^{2}}\left|A_{0}(\rho, \Phi)\right|^{2} G_{0}(\rho, \Phi) \text {. }
$$

Although $H_{01}(\rho, \Phi)$ is not separable, we can precompute $w_{0}(\Phi)$ and tabulate $G_{0}(\rho, \Phi)$ once for all pixels for coarsely sampled $\Phi$. Accurately computing $G_{0}(\rho, \Phi)$ is crucial, therefore, finely sampled $\varphi$ is necessary in (33).

2) Type II Separable Form: We can simplify further by using the sifting property of the Dirac impulse

$$
\int_{0}^{2 \pi} w_{0}(\varphi) S_{\varphi}(\rho, \Phi) \mathrm{d} \varphi \approx \frac{2}{|\rho|} w_{0}(\Phi)\left|A_{0}(\rho, \Phi)\right|^{2} .
$$

Because typically $A_{0}(\nu, \varphi)$ varies slowly, we also consider the following further approximation:

$$
A_{0}(\nu, \varphi) \approx A_{0}(0, \varphi) \text {. }
$$




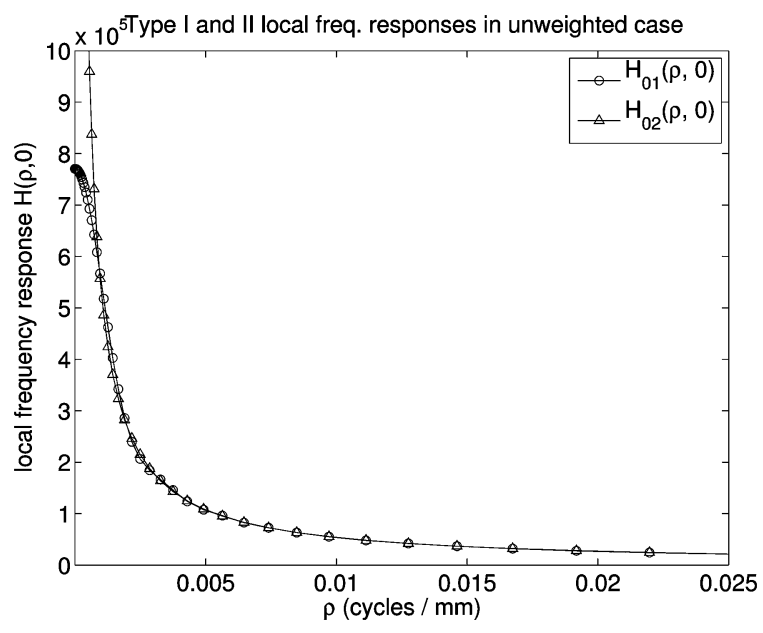

Fig. 3. Type I and Type II local frequency responses $H_{01}(\rho, 0)$ and $H_{02}(\rho, 0)$ for $\vec{n}_{0}$ at image center in unweighted case: $w(s, \beta)=1 . H_{02}(0,0)$ is not shown because it is infinite.

Combining all the above approximations yields the following separable approximation to the local frequency response:

$$
H_{0}(\rho, \Phi) \approx H_{02}(\rho, \Phi) \triangleq \frac{2\left|A_{0}(0, \varphi)\right|^{2}}{\Delta_{\beta} \Delta_{s} \Delta^{2}} \frac{w_{0}(\Phi)}{|\rho|} .
$$

This "Type II" separable form agrees with the familiar FT of $1 / r$. Fig. 3 shows the profiles of two types of local frequency responses for $\vec{n}_{0}$ at image center in unweighted case. We can see that two profiles agrees with each other closely. The discrepancy is mainly at low frequencies.

\section{QUADRATIC REGULARIZATION: $R_{0}(\rho, \Phi)$}

To evaluate the variance using (18) and (19), we also need the local frequency response of quadratic regularization, $R_{0}(\rho, \Phi)$, [7], [8], [18], [19].

Practical regularization methods are based on the differences between neighboring pixel values. For a discrete-space 2-D object $\mu[\vec{n}]$, a typical quadratic roughness penalty is given in (4) and (5) for first-order differences. The $r_{l}[\vec{n}]$ values are possibly space variant. For the purpose of local frequency response analysis, we examine the characteristics of $R(\boldsymbol{\mu})$ near a pixel $\vec{n}_{0}$ of interest, so we define $r_{l, 0} \triangleq r_{l}\left[\vec{n}_{0}\right]$ assuming $r_{l}[\vec{n}]$ values vary smoothly. Then, the quadratic roughness penalty near a pixel $\vec{n}_{0}$ has the following form:

$$
R(\boldsymbol{\mu})=\sum_{\vec{n}} \sum_{l=1}^{L} r_{l, 0} \frac{1}{2}\left(\left(c_{l} * * \mu\right)[\vec{n}]\right)^{2} .
$$

The $r_{l, 0}$ values are design parameters that affect the directionality of the regularization and hence the shape of the PSF. Each $c_{l}[\vec{n}]$ is a (typically) high-pass filter. For a first-order difference

$$
c_{l}[\vec{n}]=\xi_{l}\left(\delta_{2}[\vec{n}]-\delta_{2}\left[\vec{n}-\vec{m}_{l}\right]\right)
$$

or for a second-order difference

$$
c_{l}[\vec{n}]=\xi_{l}\left(\delta_{2}[\vec{n}]-\delta_{2}\left[\vec{n}-\vec{m}_{l}\right]\right) * * \xi_{l}\left(\delta_{2}[\vec{n}]-\delta_{2}\left[\vec{n}-\vec{m}_{l}\right]\right)
$$

where $\xi=\left\|\vec{m}_{l}\right\|^{-v / 2}, \vec{m}_{l}=\left(n_{l}, m_{l}\right)$ denotes the spatial offsets to the neighboring pixels, and $v$ is the power of weights for diagonal neighbors that can be chosen by the user. For example, common practice chooses $v=1$ [20], [21].

Applying Parseval's theorem, we can rewrite $R(\boldsymbol{\mu})$ as follows:

$$
R(\boldsymbol{\mu})=\sum_{l=1}^{L} \int_{-\pi}^{\pi} \int_{-\pi}^{\pi} \frac{1}{2} r_{l, 0}\left|C_{l}(\vec{\omega}) U(\vec{\omega})\right|^{2} \frac{\mathrm{d} \vec{\omega}}{(2 \pi)^{2}}
$$

where $\mu[\vec{n}] \stackrel{D S F T}{\longleftrightarrow} U(\vec{\omega})$ and the DSFT of a $\Lambda$-order (where $\Lambda \in \mathbb{N}$ ) difference has the following magnitude:

$$
\begin{aligned}
\left|C_{l}(\vec{\omega})\right| & =\xi_{l}^{\Lambda}\left|1-e^{-i\left(\vec{\omega} \cdot \vec{m}_{l}\right)}\right|^{\Lambda} \\
& =\xi_{l}^{\Lambda} 2^{\Lambda}\left|\sin ^{\Lambda}\left(\frac{1}{2}\left(\vec{\omega} \cdot \vec{m}_{l}\right)\right)\right| .
\end{aligned}
$$

In the polar coordinates of (19):

$$
\left|C_{l}(\rho, \Phi)\right|^{2}=\left|C_{l}\left(2 \pi \rho \Delta \vec{e}_{\Phi}\right)\right|^{2}=\xi_{l}^{2 \Lambda} 4^{\Lambda} \sin ^{2 \Lambda}\left(\pi \Delta \rho \vec{e}_{\Phi} \cdot \vec{m}_{l}\right) .
$$

Thus, the Type I local frequency response for the regularization operator is

$$
\begin{aligned}
R_{0}(\rho, \Phi) & =R_{01}(\rho, \Phi)=\sum_{l=1}^{L} r_{l, 0}\left|C_{l}(\rho, \Phi)\right|^{2} \\
& =\sum_{l=1}^{L} r_{l, 0} \xi_{l}^{2 \Lambda} 4^{\Lambda} \sin ^{2 \Lambda}\left(\pi \Delta \rho \vec{e}_{\Phi} \cdot \vec{m}_{l}\right) .
\end{aligned}
$$

Applying the approximation $\sin (x) \approx x$ to (39) yields

$$
\begin{aligned}
\left|C_{l}(\rho, \Phi)\right|^{2} & \approx \xi_{l}^{2 \Lambda}\left(\vec{m}_{l} \cdot \vec{e}_{\Phi}\right)^{2 \Lambda}(2 \pi \Delta \rho)^{2 \Lambda} \\
& =(2 \pi \rho \Delta)^{2 \Lambda} \xi_{l}^{(1-2 / v) 2 \Lambda} \cos ^{2 \Lambda}\left(\Phi-\varphi_{l}\right)
\end{aligned}
$$

where the angle between the $l$ th neighbors is

$$
\varphi_{l} \triangleq \tan ^{-1} \frac{m_{l}}{n_{l}}
$$

With this simplification, the Type II local frequency response of the regularizer is approximately separable in $(\rho, \Phi)$

$$
R_{0}(\rho, \Phi) \approx R_{02}(\rho, \Phi)=(2 \pi \rho \Delta)^{2 \Lambda} \tilde{R}_{0}(\Phi)
$$

where

$$
\tilde{R}_{0}(\Phi) \triangleq \sum_{l=1}^{L} \xi_{l}^{(1-2 / v) 2 \Lambda} r_{l, 0} \cos ^{2 \Lambda}\left(\Phi-\varphi_{l}\right)
$$

This separable form agrees with the familiar FT of the differentiation operation.

\section{VARIANCE PREDICTION IMPLEMENTATION}

Having obtained the approximations to $H_{0}(\rho, \Phi)$, the local frequency response of the Gram operator given in (35) and (37), and to $R_{0}(\rho, \Phi)$, the local frequency response of the regularizer given in (40) and (41), we can discretize the integral (18) again 
to compute the variance image. There are two variance prediction expressions for fan-beam transmission tomography based on the Type I $H_{01}(\rho, \Phi)$ given in (35) and $R_{01}(\rho, \Phi)$ given in (40), and the Type II $H_{02}(\rho, \Phi)$ given in (37) and $R_{02}(\rho, \Phi)$ given in (41).

\section{A. Double Integral Approach}

The variance prediction using $H_{01}(\rho, \Phi)$ in (35) and $R_{01}(\rho, \Phi)$ in (40) involves a double integral and can be implemented by a double summation. We call this prediction the double integral (DI) approach. The location-dependent weighting function $w_{0}(\Phi)$ can be precomputed, with the computation equivalent to one back-projection. We can coarsely sample $\Phi$ because $P_{0}(\rho, \Phi)$ is fairly smooth along $\Phi$.

\section{B. Single Integral Approach}

The separability properties of $H_{02}(\rho, \Phi)$ in (37) and $R_{02}(\rho, \Phi)$ in (41) enable us to carry out the inner integral over $\rho$ analytically. Therefore, the double-integral in (18) is reduced to one single integral over $\Phi$. Substituting (37) and (41) into (18) yields the remarkably simple expression

$\operatorname{Var}\left\{\hat{\mu}\left[\vec{n}_{0}\right]\right\} \approx \int_{0}^{2 \pi} \frac{\frac{\zeta(\Phi)}{3}}{2\left|A_{0}(0, \Phi)\right|^{2} w_{0}(\Phi)+\alpha 4 \pi^{2} \zeta(\Phi) \tilde{R}_{0}(\Phi)} \mathrm{d} \Phi$

where $\zeta(\Phi) \triangleq \rho_{\max }^{3}(\Phi) \Delta_{\beta} \Delta_{s} \Delta^{4}$. We call this prediction the single integral (SI) approach. We evaluate this integral using a finite summation, with $w_{0}(\Phi)$ and $\tilde{R}_{0}(\Phi)$ precomputed. Therefore, computing (42) is equivalent to one back-projection.

\section{Implementation of the Single Integral Prediction}

We found empirically that the SI approach (42) gave predictions that could be improved by including a single global scale factor, presumably because the SI approach (42) uses many approximations to achieve its simple form. In particular, we found that the SI method underestimates the variance, presumably because the "Fisher information" implied by Type II local frequency response in (37) is too large for low spatial frequencies. To determine the scale factor, we assumed that the DFT-based approach and the analytical approach should produce equivalent results at the image center. Specifically, we used the predicted variance for unweighted least squares estimator with standard quadratic penalty (QPULS) for unit variance data.

For QPULS estimator for unit variance data, the statistical weighting, $w(s, \beta)$ becomes 1 . Consider the standard quadratic penalty with first-order $(\Lambda=1)$ differences and second-order neighborhood $(L=4)$, for which $\varphi_{1,2,3,4}=0, \pi / 2, \pi / 4$, and $-\pi / 4$ and $r_{l, 0}=(1,1,1,1)$. We used $v=1$ both in calibration and reconstruction as is the common practice in quadratic regularization. For these choices, the Type II $R_{02}(\rho, \Phi)$ in (41) becomes independent of $\Phi$

$$
\tilde{R}_{0}(\Phi)=\sum_{l=1}^{L}\left\|\vec{m}_{l}\right\|=1+\sqrt{2} .
$$

Finally, to determine the scale factor, we computed the ratio of the variance predicted by the DFT approach over that predicted by (42). For the parameters used in our simulations, this factor was $(1.13)^{2}$. This value would need to recomputed for different system geometries or regularization parameters, but is otherwise patient independent.

\section{SimULATION RESULTS}

To evaluate the performance of the proposed methods, we implemented the variance predictions for fan-beam tomographic images reconstructed by quadratically penalized likelihood algorithm. We simulated 1000 realizations of fan-beam transmission scans using a $256 \times 256$ Zubal phantom [22] and a blank scan of $10^{6}$ counts/detector. The corresponding sinogram size was 444 samples in $s$, spaced by $\Delta_{s} \approx 2 \mathrm{~mm}$ and 492 source positions over $360^{\circ}$. We simulated the geometry of the GE LightSpeed Pro CT scanner with the source-to-detector distance around $949 \mathrm{~mm}$, the isocenter-to-detector distance 408 $\mathrm{mm}$ and $\Delta=500 / 256 \mathrm{~mm}$. We used distance-driven (DD) projectors developed by DeMan et al. [34].

An elliptical support was used for $\mathcal{S}$, with $p=43892$. For simplicity, in (34) we used the width of the central profile through the FOV

$$
d_{0}(\varphi) \approx d(\varphi) \triangleq \frac{2 z_{1} z_{2}}{\sqrt{z_{1}^{2} \sin ^{2} \varphi+z_{2}^{2} \cos ^{2} \varphi}}
$$

where $z_{1}=244.1 \mathrm{~mm}$ and $z_{2}=220.7 \mathrm{~mm}$ are the semi-major and semi-minor axes of the ellipse. This approximation is exact when $\vec{n}_{0}$ is at the ellipse center. The approximation to $\mathrm{d}(\varphi)$ becomes less accurate as $\vec{x}_{c}\left[\vec{n}_{0}\right]$ approaches the edge of the ellipse support.

For simplicity, we use $\rho_{\max }(\Phi) \approx 1 /(2 \Delta)$ and model the detector response ${ }^{2}$ in (23) by a shift-invariant rectangle of width $\Delta_{s}$

$$
b(s)=\frac{1}{\Delta_{s}} \operatorname{rect}\left(\frac{s}{\Delta_{s}}\right) .
$$

In the case of a square pixel basis $\chi(\vec{x})=\operatorname{rect}_{2}(\vec{x})^{3}$, we have from (52) (see Appendix I)

$$
A_{0}(\nu, \varphi)=\operatorname{sinc}\left(\frac{\Delta_{s} \nu}{m_{0}(\varphi)}\right) \Delta^{2} \operatorname{sinc}(\nu \Delta \cos \varphi) \operatorname{sinc}(\nu \Delta \sin \varphi)
$$

which we substitute into (32). In our simulation, we make the following simplification:

$$
m_{0}(\varphi) \approx m_{c}(\varphi)=m_{c}=\frac{D_{\mathrm{sd}}}{D_{\mathrm{s} 0}}
$$

where $m_{c}$ is the value of $m_{0}(\varphi)$ at the image center.

We chose the regularization parameter $\alpha=2^{11}$ to give full - widthathalf - maximum $=1.72$ pixels, i.e., 3.4 $\mathrm{mm}$, at the center of the image. For each realization, $\hat{\boldsymbol{\mu}}$ was reconstructed using 70 iterations of the convergent incremental optimization transfer algorithm (PL-IOT) [23] with 41 subsets and no nonnegativity constraint. The initial images were the filtered back-projection (FBP) images with equivalent spatial resolution, obtained by post-filtering ramp-filtered FBP images

\footnotetext{
${ }^{2} \mathrm{~A}$ more accurate model could include detector deadspace and crosstalk effects.

${ }^{3} \operatorname{rect}_{2}(\vec{x}) \triangleq \operatorname{rect}(x) \operatorname{rect}(y)$ is a 2-D square function.
} 


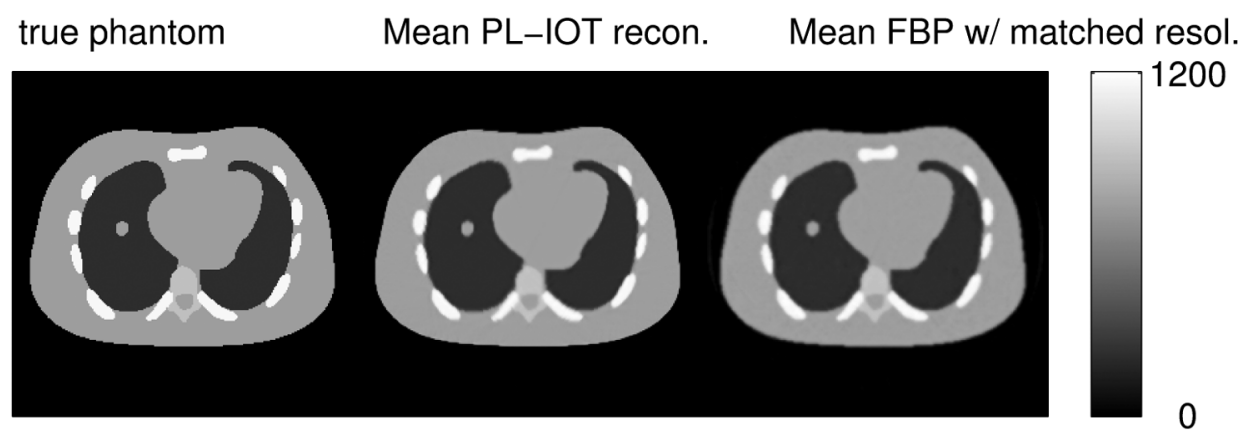

predicted std. dev. $\quad$ PL-IOT empirical $\quad$ FBP empirical (divided by 1.8 )
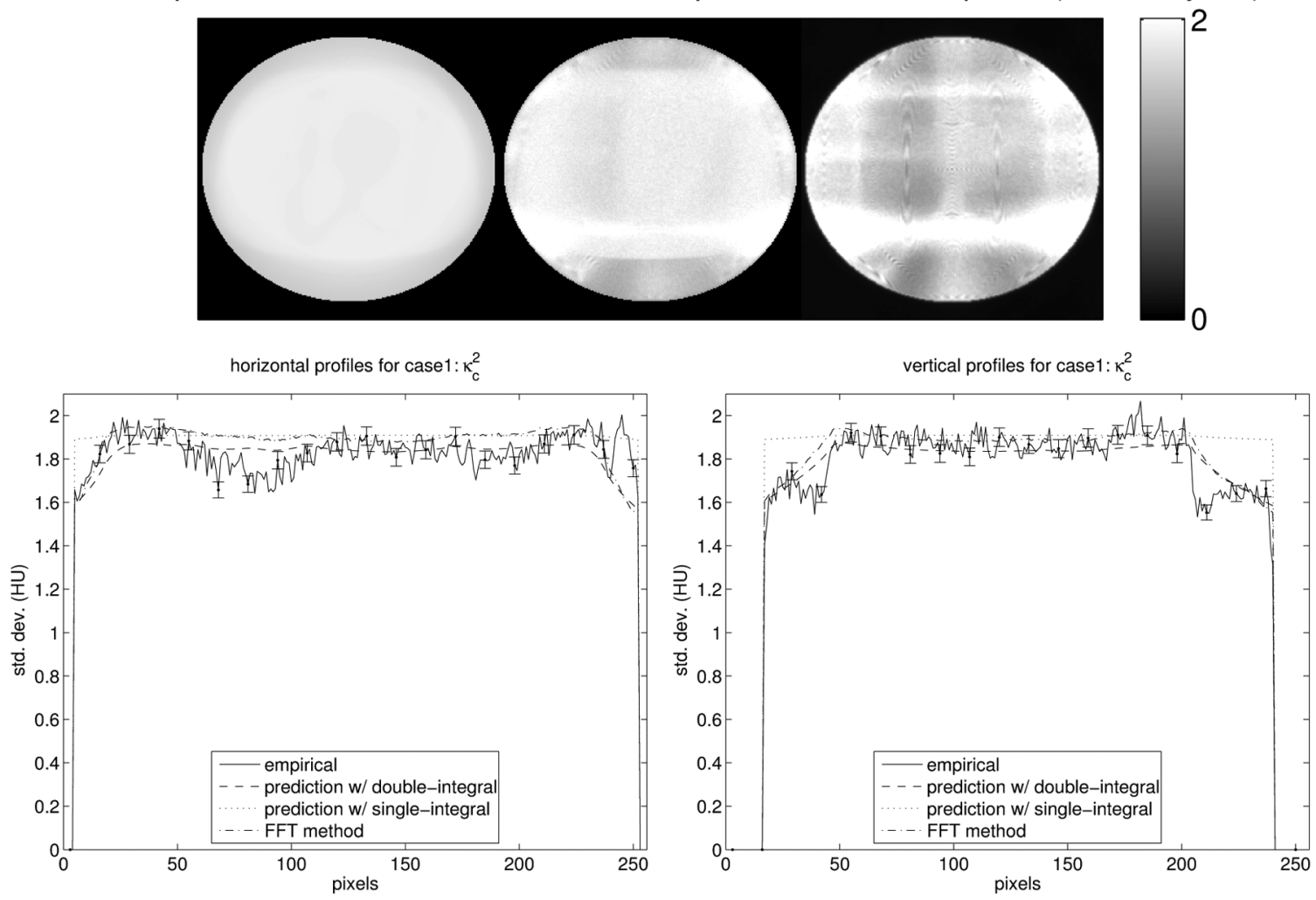

Fig. 4. Predicted and empirical standard deviation images (in HU) and central profiles for Zubal phantom for PL fan-beam transmission image reconstruction using the standard quadratic penalty: $\tilde{R}_{0}=(1+\sqrt{2}) \kappa_{c}^{2}$.

with the designed PSF. We computed the sample standard deviation pixel by pixel within the finite support $\mathcal{S}$ used in reconstruction. All images and profiles are shown in Hounsfield unit (HU).

Two prediction approaches are investigated here: the DI approximation (18) with Type I $H_{01}(\rho, \Phi)$ in (35) and $R_{01}(\rho, \Phi)$ in (40), and the SI approximation (42) with $\tilde{R}_{0}(\Phi)$ in (41). The former formula was derived with fewer approximations while the latter formula involves more approximations. The accuracy and computation time are compared below.

We considered two types of regularization: standard and certainty-based [24]. In both cases, we implemented (40) and (41) for regularization with first-order $(\Lambda=1)$ differences and second-order neighborhood $(L=4)$. In both cases, the standard deviation images predicted by the DI approach are displayed while both DI and SI predictions are compared in the profile plots.

\section{A. Standard Quadratic Penalty Function}

We first considered a standard quadratic penalty where

$$
r_{l, 0}=\kappa_{c}^{2}
$$

and $\kappa_{c}^{2}$ is the value of $\kappa^{2}\left[\vec{n}_{0}\right]$ at the image center in (46). This choice assures that the resolution of the two studies is matched at the image center. In this case, $\tilde{R}_{0}(\Phi)=(1+\sqrt{2}) \kappa_{c}^{2}$ is a constant for all pixels.

Fig. 4 shows the reconstructed images and empirical standard deviation images. The empirical standard deviation image for FBP is also shown. The average of FBP standard deviations 


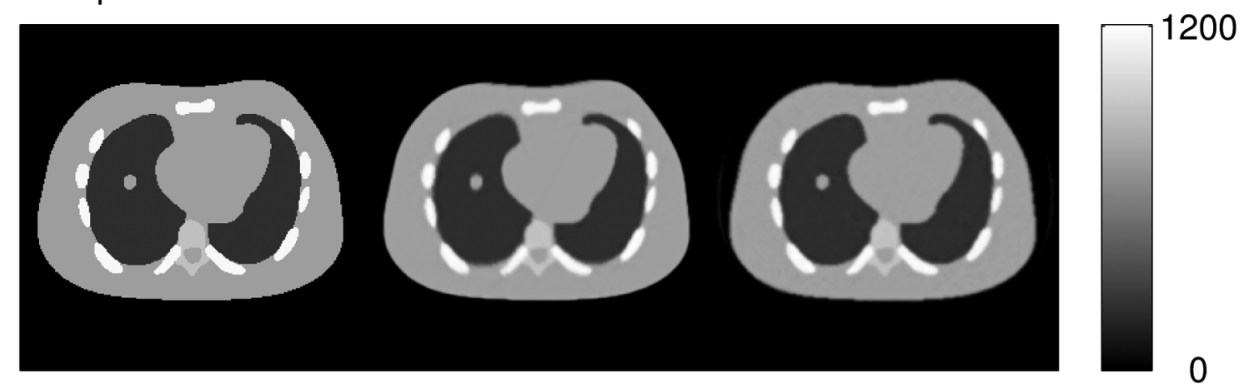

predicted std. dev. $\quad$ PL-IOT empirical $\quad$ FBP empirical (divided by 3)
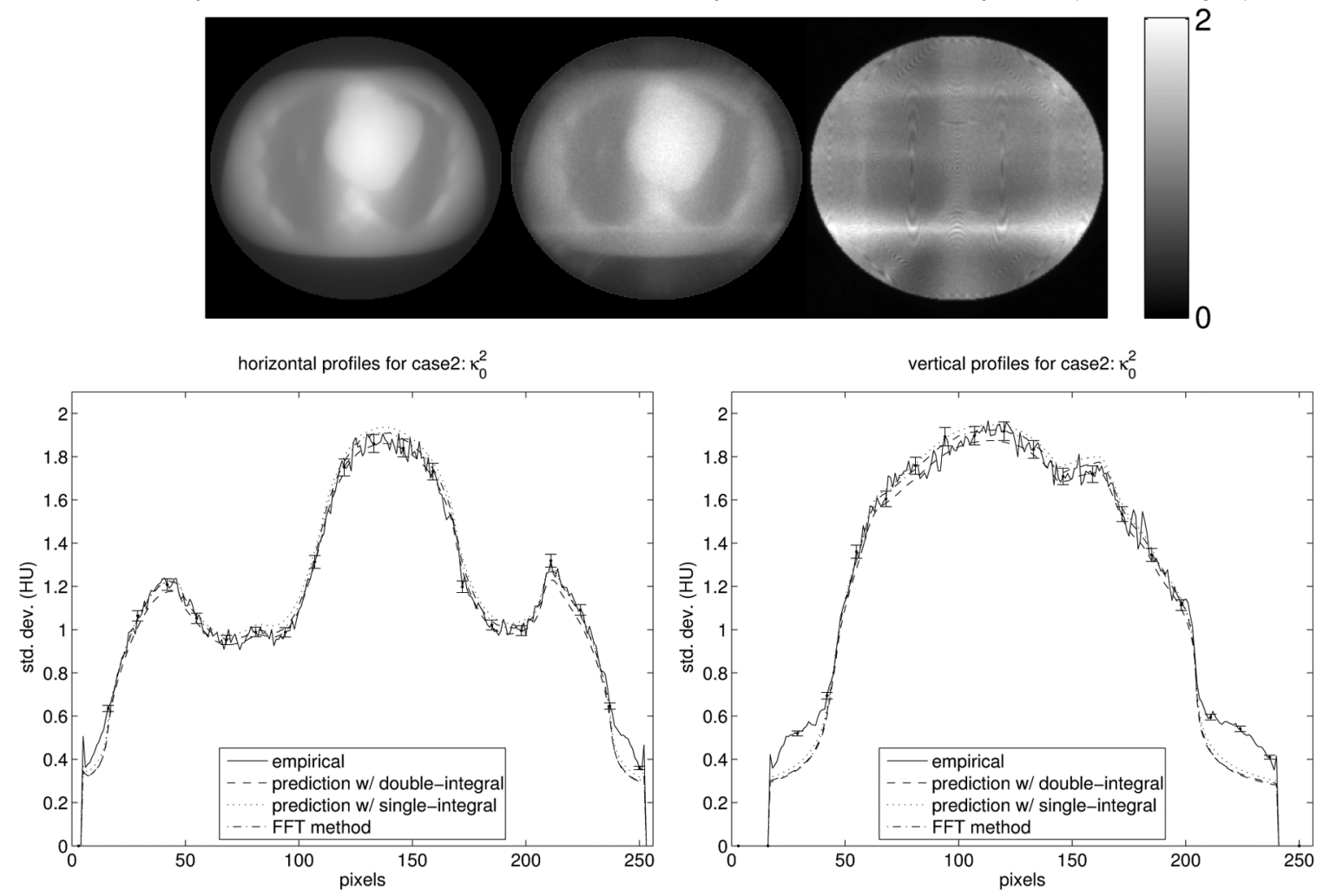

Fig. 5. Predicted and empirical standard deviation images (in HU) and central profiles for Zubal phantom for PL fan-beam transmission image reconstruction using the certainty-based quadratic penalty.

is around $2.2 \mathrm{HU}$, approximately 1.8 times higher than that of PL-IOT, 1.2 HU, illustrating the noise advantage of the statistical reconstruction methods at matched resolution.

Fig. 4 also shows the central horizontal and vertical profiles of the standard deviation maps. The analytical, the FFT-based and the empirical standard deviations agree with one another very closely within the object. The largest discrepancy within the object was about $10 \%$ in the left lung for unknown reasons.

\section{B. Certainty-Based Quadratic Penalty Function}

We next investigate a more complicated regularizer that was designed to achieve nearly uniform spatial resolution [24]. In this case, we used space-varying regularizer

$$
r_{l, 0}=\kappa^{2}\left[\vec{n}_{0}\right]
$$

where

$$
\kappa^{2}\left[\vec{n}_{0}\right] \triangleq \frac{1}{2 \pi} \int_{0}^{2 \pi} w\left(s\left(r_{0}(\varphi)\right), \beta\left(r_{0}(\varphi), \varphi\right)\right) \mathrm{d} \varphi .
$$

Here, $\tilde{R}_{0}(\Phi)$ is still independent of $\Phi$, but varies spatially. Computing the "certainty map" (46) requires a simple backprojection with fan-to-parallel beam rebinning.

Fig. 5 shows the reconstructed images, standard deviation images and central horizontal and vertical profiles. In this case, the average of FBP standard deviations is around $2.2 \mathrm{HU}$, approximately 1.8 times higher than that of PL-IOT, 0.7 HU. The analytical, the FFT-based and the empirical standard deviations agree with one another very closely within the object.

In both cases, the analytical and the FFT-based predictions are somewhat less accurate near the edge of the finite support used in image reconstruction. This is probably due to the fact 
that the "local stationarity" approximation is less accurate in this area where the statistical weights $w(s, \beta)$ can vary rapidly. The approximation (44) may also vary rapidly in our study, so it may be possible to improve accuracy near the edges of the FOV by using $d_{0}(\varphi)$.

\section{Computation Time and Accuracy}

In our calculations, we used 123 samples in $\Phi$ and 128 samples in $\rho$ in (18) to predict a $256 \times 256$ standard deviation image. Both DI and SI predictions precompute $w_{0}(\Phi)$ and $G_{0}(\rho, \Phi)$. The precomputation time for $w_{0}(\Phi)$ was about 19 $\mathrm{s}$ on dual Intel Xeon $3.40 \mathrm{GHz}$ CPU. The precomputation time for $G_{0}(\rho, \Phi)$ with 492 samples in $\varphi$ was $2.3 \mathrm{~s}$. The DI prediction requires no scale factor precomputation and the computation time was about $135 \mathrm{~s}$. The SI prediction requires the scale factor precomputation that is $(1.13)^{2}$ in our case, and the computation time for prediction was about $0.6 \mathrm{~s}$. In contrast, the FFT-based prediction needed about 374 s to compute only one single central profile. As expected, the DI prediction is slightly more accurate than the SI prediction, particularly near edges. The SI prediction matches a bit better with the FFT-based prediction because the scale factor calibration was based on FFT-predicted values. For the purposes of regularization design or noise exploration, we believe that the very fast SI approach is adequate.

Because we only compute two central profiles of the FFTbased prediction in each case, we compute the normalized rootmean square (NRMS) percent errors only for these two central profiles. For $\kappa_{c}^{2}$ case, the NRMS percent errors for FFT, DI and SI are $6.6 \%, 6.8 \%$, and $6.6 \%$; for $\kappa_{0}^{2}$ case, the NRMS percent errors for FFT, DI and SI are 6.5\%, 6.0\%, and 8.3\%, respectively.

\section{CONCLUSION AND DISCUSSION}

This paper has developed analytical variance approximations for 2-D tomography. The double integral (18) with (35) and (40), and the single integral (42) provide fast and accurate variance predictions for a quadratically penalized likelihood estimator in fan-beam tomography. The simplest of the proposed approaches (42) requires one backprojection with some additional summations, which is much less computation than previous FFT-based methods. In fact, using the proposed methods, we can predict the variance map in much less time than it takes to reconstruct a single image. The proposed approximations are especially useful in the case that the variance information is needed for many or all pixels, such as when choosing space-varying regularization parameters [6]. The empirical results from the simulated fan-beam CT transmission scans demonstrate that the proposed variance approximations are very accurate. Future work will explore using these predictions for regularization design.

Although we focused on variance prediction, by using (15) we could also easily predict covariances and thus predict the covariance of a region of interest whose size is small enough that the local approximations are sufficiently accurate. However, if only a single local autocorrelation function is needed, then the FFT approach is probably easier to use. For analysis of detectability of lesions with unknown locations, autocorrelations at many spatial positions may be needed [10], [25]-[28], in which case the proposed approach based on (15) can save computation. The matrix method described in (6) and (7) is also applicable to other imaging modalities, such as positron emission tomography (PET) and single-photon-emission computed tomography (SPECT) [1]. Therefore, the proposed methods are also readily extended to those imaging modalities, with different considerations of the weighting function.

The proposed analytical variance approximations are only investigated in the case of the shift-invariant detector blur. We can also generalize the analysis to shift-variant detector blur where the local shift-invariance approximation is applicable, e.g., for varifocal collimators in SPECT. In this case, $b\left(s-s^{\prime}\right)$ is replaced by $b\left(s, s^{\prime}\right)$ in $(24)$ and $b_{0}(r, \varphi)$ in $(53)$ becomes

$$
\begin{aligned}
& b_{0}(r, \varphi) \triangleq m_{0}(\varphi) b\left(s_{0}(\varphi)+m_{0}(\varphi) r, s_{0}(\varphi)\right) \\
& \text { where } \\
& s_{0}(\varphi) \triangleq D_{\mathrm{sd}} \arcsin \frac{r_{0}(\varphi)}{D_{\mathrm{s} 0}}
\end{aligned}
$$

This paper has focused on 2-D fan-beam geometry. Three-dimensional generalization of these methods can be done by applying the same principles [29]. This paper has also focused on analytical variance approximations for the case of quadratic regularization. An interesting challenge for future work is to generalize the analysis to the case of edge-preserving nonquadratic regularization. The analysis in [30] may be a useful starting point.

\section{APPENDIX I}

DERIVATION OF LOCAL IMPULSE RESPONSE

Reparameterize variables $s$ and $\beta$ in (27) according to the inversion of (20) and (21)

$$
\begin{aligned}
s \rightarrow s(r) & =D_{\mathrm{sd}} \arcsin \left(\frac{r}{D_{\mathrm{s} 0}}\right) \\
\beta \rightarrow \beta(r, \varphi) & =\varphi-\arcsin \left(\frac{r}{D_{\mathrm{s} 0}}\right) .
\end{aligned}
$$

Then the fan-to-parallel beam rebinning of $a(s, \beta ; \vec{n})$ is

$$
\begin{aligned}
a(s(r), \beta(r, \varphi) ; \vec{n}) \approx & \int b\left(s(r)-s\left(r^{\prime}\right)\right) \Delta \\
& \cdot g\left(\frac{r^{\prime}-r_{\varphi}[\vec{n}]}{\Delta}, \varphi\right)\left|\dot{s}\left(r^{\prime}\right)\right| \mathrm{d} r^{\prime} \\
& \triangleq a(r, \varphi ; \vec{n})
\end{aligned}
$$

because $r\left(s\left(r^{\prime}\right)\right)=r^{\prime}$ and $\varphi\left(s\left(r^{\prime}\right), \beta(r, \varphi)\right) \approx \varphi$ for $r \approx r^{\prime}$.

A first-order Taylor expansion of $s(r)$ around $r^{\prime}$ yields

$$
s(r)-s\left(r^{\prime}\right) \approx \dot{s}\left(r^{\prime}\right)\left(r-r^{\prime}\right)
$$

Substituting into (47), the system matrix elements become

$$
\begin{aligned}
& a(r, \varphi ; \vec{n}) \approx \int b\left(\dot{s}\left(r^{\prime}\right)\left(r-r^{\prime}\right)\right) \Delta \\
& \quad \cdot g\left(\frac{r^{\prime}-r_{\varphi}[\vec{n}]}{\Delta}, \varphi\right)\left|\dot{s}\left(r^{\prime}\right)\right| \mathrm{d} r^{\prime}
\end{aligned}
$$




$$
\begin{aligned}
\breve{h} \varphi\left[\vec{n} ; \vec{n}^{\prime}\right] & \triangleq \int_{-\infty}^{\infty} a\left(r-r_{\varphi}[\vec{n}], \varphi ; \vec{n}\right) a\left(r-r_{\varphi}\left[\vec{n}^{\prime}\right], \varphi ; \vec{n}^{\prime}\right) \mathrm{d} r \\
\breve{w}\left(\varphi ; \vec{n} ; \vec{n}^{\prime}\right) & \triangleq \frac{\int_{-\infty}^{\infty} \bar{w}(r, \varphi)|J(r)| a\left(r-r_{\varphi}[\vec{n}], \varphi\right) a\left(r-r_{\varphi}\left[\vec{n}^{\prime}\right], \varphi\right) \mathrm{d} r}{\int_{-\infty}^{\infty} a\left(r-r_{\varphi}[\vec{n}], \varphi\right) a\left(r-r_{\varphi}\left[\vec{n}^{\prime}\right], \varphi\right) \mathrm{d} r} \\
\bar{w}(r, \varphi) & \triangleq w(s(r), \beta(r, \varphi)) .
\end{aligned}
$$

Substituting (48) into (27) and changing variables from $(s, \beta)$ to $(r, \varphi)$ using $(20)$ and $(21)$ yields the local impulse approximation

$$
\begin{aligned}
\breve{h}_{\mathrm{d}}\left[\vec{n} ; \vec{n}^{\prime}\right] \approx & \frac{1}{\Delta_{\beta}} \frac{1}{\Delta_{s}} \int_{0}^{2 \pi} \int_{-\infty}^{\infty} \bar{w}(r, \varphi) a(r, \varphi ; \vec{n}) a\left(r, \varphi ; \vec{n}^{\prime}\right) \\
& \cdot|J(r)| \mathrm{d} r \mathrm{~d} \varphi \\
= & \frac{1}{\Delta_{\beta}} \frac{1}{\Delta_{s}} \int_{0}^{2 \pi} \breve{w}\left(\varphi ; \vec{n} ; \vec{n}^{\prime}\right) \breve{h} \varphi\left[\vec{n} ; \vec{n}^{\prime}\right] \mathrm{d} \varphi
\end{aligned}
$$

where $|J(r)|=|\dot{s}(r)|$ is the determinant of Jacobian matrix, and see equation (50) at the top of this page.

Let $r_{0}(\varphi) \triangleq r_{\varphi}\left[\vec{n}_{0}\right]$. Because $\dot{s}(r)$ is fairly smooth, we make the following approximation for $r^{\prime} \approx r_{0}(\varphi)$ :

$$
\dot{s}\left(r^{\prime}\right) \approx \dot{s}\left(r_{0}(\varphi)\right) \triangleq m_{0}(\varphi) .
$$

Substituting (51) into (48) and simplifying yields

$$
\begin{aligned}
a(r, \varphi ; \vec{n}) \approx & a\left(r, \varphi ; \vec{n}_{0}\right) \triangleq a_{0}(r, \varphi) \\
= & \int b_{0}\left(r-r_{\varphi}[\vec{n}]-r^{\prime \prime}, \varphi\right) \\
& \cdot \Delta g\left(\frac{r^{\prime \prime}}{\Delta}, \varphi\right) \mathrm{d} r^{\prime \prime}
\end{aligned}
$$

with $r^{\prime \prime}=r^{\prime}-r_{\varphi}[\vec{n}]$ and

$$
b_{0}(r, \varphi) \triangleq m_{0}(\varphi) b\left(m_{0}(\varphi) r\right) .
$$

Therefore, we further simply (49) as follows:

$$
\breve{h} \breve{\mathrm{d}}\left[\vec{n} ; \vec{n}^{\prime}\right] \approx \frac{1}{\Delta_{\beta}} \frac{1}{\Delta_{s}} \int_{0}^{2 \pi} w_{0}(\varphi) \breve{h}_{0}\left(\Delta\left(\vec{n}-\vec{n}^{\prime}\right) \cdot \vec{e}_{\varphi}, \varphi\right) \mathrm{d} \varphi
$$

where because $\bar{w}(r, \varphi)$ often varies slowly in $r$ relative to the typically sharp peak of $a_{0}(r, \varphi)$ at $r=0$

$$
\begin{aligned}
\breve{h}_{0}(r, \varphi) & \triangleq a_{0}(r, \varphi) \star a_{0}(r, \varphi), \\
\breve{w}\left(\varphi ; \vec{n} ; \vec{n}^{\prime}\right) & \approx \frac{\int_{-\infty}^{\infty} \bar{w}(r, \varphi)\left|m_{0}(\varphi)\right| a_{0}^{2}\left(r-r_{\varphi}\left[\vec{n}_{0}\right], \varphi\right) \mathrm{d} r}{\int_{-\infty}^{\infty} a_{0}^{2}\left(r-r_{\varphi}\left[\vec{n}_{0}\right], \varphi\right) \mathrm{d} r} \\
& \approx\left|m_{0}(\varphi)\right| \bar{w}\left(r_{0}(\varphi), \varphi\right) \triangleq w_{0}(\varphi)
\end{aligned}
$$

where $\star$ denotes a 1-D autocorrelation with respect to $r$.

\section{APPENDIX II}

DERIVATION OF LOCAL FREQUENCY RESPONSE

The simplest approach to finding the local frequency response would be to take the 2-D Fourier transform of the local impulse response in (28), while ignoring the "edge effects" caused by the support functions in (25). We found this approach to yield suboptimal accuracy. One way to consider the edge effects is to use a triangular function with the angular-dependent width

$$
\tilde{h}_{\varphi}\left[\vec{n} ; \vec{n}_{0}\right] \triangleq \breve{h}_{0}\left(\Delta \vec{n} \cdot \vec{e}_{\varphi}, \varphi\right) \eta_{1}(\Delta \vec{n})
$$

where

$$
\eta_{1}(\vec{x}) \triangleq \operatorname{tri}\left(\frac{\vec{x} \cdot \vec{e}_{\varphi}^{\perp}}{d_{0}(\varphi)}\right)
$$

and $d_{0}(\varphi)$ denotes the length of the chord through $\vec{n}_{0}$ through the FOV at angle $(\varphi+\pi / 2)$. This approach is inspired by circulant approximations for Toeplitz matrices [13], [31], [32], preserving the nonnegative definite property of $\boldsymbol{A}^{\prime} \boldsymbol{W} \boldsymbol{A}$. This choice might not be optimal in our application, and further investigation may be beneficial.

One alternative way to consider edge effects is to use the coordinate transformation recommended for analyzing quasi-stationary noise in $[12$, p. 870$]$ as follows:

$$
\begin{aligned}
\tilde{h}_{\varphi}\left[\vec{n} ; \vec{n}_{0}\right] & \triangleq h_{\varphi}\left[\vec{n}_{0}+\frac{\vec{n}}{2} ; \vec{n}_{0}-\frac{\vec{n}}{2}\right] \\
& =\breve{h}_{0}\left(\Delta \vec{n} \cdot \vec{e}_{\varphi}, \varphi\right) \eta_{2}(\Delta \vec{n})
\end{aligned}
$$

where $\vec{x}_{0} \triangleq \vec{x}_{c}\left[\vec{n}_{0}\right]$, and $\eta_{2}$ denotes the support of the image

$$
\eta_{2}(\vec{x}) \triangleq \eta\left(\vec{x}_{0}+\frac{\vec{x}}{2}\right) \eta\left(\vec{x}_{0}-\frac{\vec{x}}{2}\right) .
$$

This approach yields a local impulse response that is symmetric in $\vec{n}$, thus ensuring that its spectrum is real.

Another alternative is to refer all displacements relative to the point $\vec{n}_{0}$ as follows:

$$
\begin{aligned}
\tilde{h}_{\varphi}\left[\vec{n} ; \vec{n}_{0}\right] & \triangleq h_{\varphi}\left[\vec{n}_{0}+\vec{n} ; \vec{n}_{0}\right] \\
& =\breve{h}_{0}\left(\Delta \vec{n} \cdot \vec{e}_{\varphi}, \varphi\right) \eta_{3}(\Delta \vec{n}),
\end{aligned}
$$

where

$$
\eta_{3}(\vec{x}) \triangleq \eta\left(\vec{x}_{0}+\vec{x}\right) \eta\left(\vec{x}_{0}\right) .
$$

This choice is not symmetric in $\vec{n}$ but it better corresponds to the local Fourier analysis based on the DFT of $\boldsymbol{A}^{\prime} \boldsymbol{W} \boldsymbol{A} \mathbf{e}_{j}$. For simplicity, we could also approximate (58) as follows:

$$
\eta_{4}(\vec{x}) \approx \eta_{0}(\vec{x})=\eta(\vec{x}) \eta\left(\vec{x}_{0}\right) .
$$

This choice also yields a local impulse response that is symmetric in $\vec{n}$ provided $\eta(\vec{x})$ is symmetric itself.

We focus on $\eta_{1}(\vec{x})$ in (56) hereafter because it preserves the property of nonnegative definiteness [33]. Define the following 
"strip like" function:

$$
s_{\varphi}(\vec{x}) \triangleq \breve{h}_{0}\left(\vec{x} \cdot \vec{e}_{\varphi}, \varphi\right) \eta_{1}(\vec{x})
$$

and $s_{\varphi}(\vec{x}) \stackrel{2 \mathrm{D} F T}{\longleftrightarrow} S_{\varphi}(u, v)$. Taking the DSFT of (54) yields the following result:

$$
H_{\mathrm{d} 0}(\vec{\omega})=\frac{1}{\Delta_{\beta}} \frac{1}{\Delta_{s}} \int_{0}^{2 \pi} w_{0}(\varphi) H_{\varphi}(\vec{\omega}) \mathrm{d} \varphi
$$

where $H_{\varphi}(\vec{\omega})$ is the spectrum of $\tilde{h}_{\varphi}\left[\vec{n} ; \vec{n}_{0}\right]=s_{\varphi}[\Delta \vec{n}]$, as follows:

$$
\begin{aligned}
H_{\varphi}(\vec{\omega}) & =\sum_{\vec{n}} s_{\varphi}(\Delta \vec{n}) e^{-\imath(\vec{\omega} \cdot \vec{n})} \\
& \approx \frac{1}{\Delta^{2}} \iint s_{\varphi}(\vec{x}) e^{-\imath(1 / \Delta)(\vec{\omega} \cdot \vec{x})} \mathrm{d} \vec{x} \\
& =\frac{1}{\Delta^{2}} S_{\varphi}\left(\frac{\vec{\omega}}{2 \pi \Delta}\right) .
\end{aligned}
$$

The 2-D FT of $s_{\varphi}(\vec{x})$ is easiest to see for the case $\varphi=0$

$$
\begin{aligned}
s_{0}(x, y) & =\breve{h}_{0}(x, 0) \operatorname{tri}\left(\frac{y}{d_{0}(0)}\right) \\
\stackrel{2 \mathrm{D} \mathrm{FT}}{\longleftrightarrow} S_{0}(u, v) & =\left|A_{0}(u, 0)\right|^{2} d_{0}(0) \operatorname{sinc}^{2}\left(d_{0}(0) v\right)
\end{aligned}
$$

where $A_{0}(\nu, \varphi)$ is associated with the detector response and basis effect, given in (45). By the rotation property of the 2-D FT

$$
\begin{aligned}
S_{\varphi}(\rho, \Phi) \approx\left|A_{0}(\rho \cos (\Phi-\varphi), \varphi)\right|^{2} \\
\cdot d_{0}(\varphi) \operatorname{sinc}^{2}\left(d_{0}(\varphi) \rho \sin (\Phi-\varphi)\right) .
\end{aligned}
$$

Therefore, using (55) and (61), the local frequency response $H_{0}(\rho, \Phi)$ around a point $\vec{n}_{0}$ is

$$
H_{0}(\rho, \Phi) \approx \frac{1}{\Delta_{\beta} \Delta_{s} \Delta^{2}} \int_{0}^{2 \pi} w_{0}(\varphi) S_{\varphi}(\rho, \Phi) \mathrm{d} \varphi .
$$

\section{REFERENCES}

[1] J. A. Fessler, "Mean and variance of implicitly defined biased estimators (such as penalized maximum likelihood): Applications to tomography," IEEE Trans. Imag. Process., vol. 5, no. 3, pp. 493-506, Mar. 1996.

[2] H. H. Barrett, D. W. Wilson, and B. M. W. Tsui, "Noise properties of the EM algorithm: I. Theory," Phys. Med. Biol., vol. 39, no. 5, pp. 833-846, May 1994.

[3] D. W. Wilson, B. M. W. Tsui, and H. H. Barrett, "Noise properties of the EM algorithm: II. Monte Carlo simulations," Phys. Med. Biol., vol. 39, no. 5, pp. 847-872, May 1994.

[4] J. Qi and R. M. Leahy, "A theoretical study of the contrast recovery and variance of MAP reconstructions from PET data," IEEE Trans. Med. Imag., vol. 18, no. 4, pp. 293-305, Apr. 1999.

[5] J. W. Stayman and J. A. Fessler, "Efficient calculation of resolution and covariance for fully-3D SPECT," IEEE Trans. Med. Imag., vol. 23, pp. 1543-1556, Dec. 2004.

[6] H. Shi and J. A. Fessler, "Quadratic regularization design for fan beam transmission tomography," in Proc. SPIE 5747, Med. Imag. 2005: Image Proc., 2005, pp. 2023-2033.

[7] J. A. Fessler, "Analytical approach to regularization design for isotropic spatial resolution," in Proc. IEEE Nucl. Sci. Symp. Med. Imag. Conf., 2003, vol. 3, pp. 2022-2026.

[8] Spatial resolution properties of penalized weighted least-squares image reconstruction with model mismatch Commun. Sign. Proc. Lab., Dept. EECS, Univ. Michigan, Ann Arbor, MI, Tech. Rep. 308, 1997 [Online]. Available: http: //www.eecs.umich.edu/ fessler

[9] J. Qi and R. H. Huesman, "Theoretical study of lesion detectability of MAP reconstruction using computer observers," IEEE Trans. Med. Imag., vol. 20, no. 8, pp. 815-822, Aug. 2001.
[10] P. Khurd and G. Gindi, "Rapid computation of LROC figures of merit using numerical observers (for SPECT/PET reconstruction)," IEEE Trans. Nucl. Sci., vol. 52, no. 3, pp. 618-626, Jun. 2005.

[11] J. Qi and R. M. Leahy, "Resolution and noise properties of MAP reconstruction for fully 3D PET," IEEE Trans. Med. Imag., vol. 19, no. 5, pp. 493-506, May 2000.

[12] H. H. Barrett and K. J. Myers, Foundations of Image Science. New York: Wiley, 2003.

[13] R. H. Chan and M. K. Ng, "Conjugate gradient methods for Toeplitz systems," SIAM Rev., vol. 38, no. 3, pp. 427-482, Sep. 1996.

[14] J. A. Fessler and S. D. Booth, "Conjugate-gradient preconditioning methods for shift-variant PET image reconstruction," IEEE Trans. Imag. Process., vol. 8, no. 5, pp. 688-699, May 1999.

[15] J. W. Stayman and J. A. Fessler, "Regularization for uniform spatial resolution properties in penalized-likelihood image reconstruction," IEEE Trans. Med. Imag., vol. 19, no. 6, pp. 601-615, Jun. 2000.

[16] H. Peng and H. Stark, "Direct Fourier reconstruction in fan-beam tomography," IEEE Trans. Med. Imag., vol. 6, no. 9, pp. 209-219, Sep. 1987.

[17] J. K. Older and P. C. Johns, "Matrix formulation of computed tomogram reconstruction," Phys. Med. Biol., vol. 38, no. 8, pp. 1051-1064, Aug. 1993

[18] M. Unser, A. Aldroubi, and M. Eden, "Recursive regularization filters: Design, properties, and applications," IEEE Trans. Pattern Anal. Mach. Intell., vol. 13, no. 3, pp. 272-277, Mar. 1991.

[19] H. Stark and E. T. Olsen, "Projection-based image restoration," J. Opt. Soc. Amer. A, vol. 9, no. 11, pp. 1914-1919, Nov. 1992.

[20] J. A. Fessler, "Penalized weighted least-squares image reconstruction for positron emission tomography," IEEE Trans. Med. Imag., vol. 13, no. 2, pp. 290-300, Jun. 1994.

[21] J. Nuyts and J. A. Fessler, "A penalized-likelihood image reconstruction method for emission tomography, compared to post-smoothed maximum-likelihood with matched spatial resolution," IEEE Trans. Med. Imag., vol. 22, no. 9, pp. 1042-1052, Sep. 2003.

[22] G. Zubal, G. Gindi, M. Lee, C. Harrell, and E. Smith, "High resolution anthropomorphic phantom for Monte Carlo analysis of internal radiation sources," in Proc. IEEE Symp. Computer-Based Med. Syst., Jun. 1990 , pp. 540-547.

[23] S. Ahn, J. A. Fessler, D. Blatt, and A. O. Hero, "Convergent incremental optimization transfer algorithms: Application to tomography," IEEE Trans. Med. Imag., vol. 25, no. 3, pp. 283-296, Mar. 2006.

[24] J. A. Fessler and W. L. Rogers, "Spatial resolution properties of penalized-likelihood image reconstruction methods: Space-invariant tomographs," IEEE Trans. Imag. Process., vol. 5, no. 9, pp. 1346-1358, Sep. 1996.

[25] P. Khurd and G. Gindi, "Fast LROC analysis of Bayesian reconstructed emission tomographic images using model observers," Phys. Med. Biol., vol. 50, no. 7, pp. 1519-1532, Apr. 2005.

[26] J. Qi and R. H. Huesman, "Fast approach to evaluate MAP reconstruction for lesion detection and localization," in Proc. SPIE 5372, Med. Imag. 2004: Image Perception, Observer Performance, Technology Assessment, 2004, pp. 273-282.

[27] P. K. Khurd and G. R. Gindi, "LROC model observers for emission tomographic reconstruction," in Proc. SPIE 5372, medical imaging 2004: Image perception, observer performance, and technology assessment, 2004, pp. 509-520.

[28] A. Yendiki and J. A. Fessler, "Analysis of unknown-location signal detectability for regularized tomographic image reconstruction," in Proc. IEEE Intl. Symp. Biomed. Imag., Apr. 2006, pp. 279-83.

[29] Y. Zhang-O'Connor and J. A. Fessler, "Fast variance predictions for 3D cone-beam CT with quadratic regularization," in SPIE Phys. Med. Imag., Apr. 2007, accepted for publication.

[30] S. Ahn and R. M. Leahy, "Spatial resolution properties of nonquadratically regularized image reconstruction for PET," in Proc. IEEE Int. Symp. Biomed. Imag., 2006, pp. 287-290.

[31] T. F. Chan, "An optimal circulant preconditioner for Toeplitz systems," SIAM J. Sci. Statist. Comp., vol. 9, no. 4, pp. 766-771, Jul. 1988.

[32] T. F. Chan and J. A. Olkin, "Circulant preconditioners for Toeplitzblock matrices," Numer. Algorithms, vol. 6, pp. 89-101, 1994.

[33] E. Tyrtyshnikov, "Optimal and superoptimal circulant preconditioners," SIAM J. Matrix. Anal. Appl., vol. 13, no. 2, pp. 459-473, 1992.

[34] B. De Man and S. Basu, "Distance-driven projection and backprojection in three dimensions," Phys. Med. Biol., vol. 49, no. 11, pp. 2463-2475, Jun. 2004. 